\title{
PERFIL DOS VISITANTES DO PARQUE NATURAL MUNICIPAL DA TAQUARA, DUQUE DE CAXIAS, RJ
}

\author{
Alexandre Takio Kitagawa ${ }^{1}$ \\ Malena Carvalho da Costa ${ }^{2}$ \\ Lucas Julião Mello de Lima \\ Mônica Gonçalves Kitagawa ${ }^{2}$ \\ Hernani Aguiar $^{3}$ \\ Rodrigo de Oliveira Lula Salles ${ }^{4}$ \\ Nathália Carina dos Santos Silva ${ }^{5}$
}

\section{Resumo}

O processo de visitação em unidades de conservação vem aumentando nos últimos anos e pode trazer benefícios sociais e econômicos, além dos riscos ambientais decorrentes de possíveis impactos negativos. O presente estudo tem como tema o Parque Natural Municipal da Taquara, município de Duque de Caxias - RJ, que faz limite ao Norte com a Área de Proteção Ambiental de Petrópolis e com a Reserva Biológica de Tinguá. Nos meses mais quentes recebe aproximadamente 4.000 visitantes por mês. Para melhoria da estrutura do local e implantação de um programa de Educação Ambiental, é necessário conhecer o perfil dos visitantes e que pode contribuir na elaboração de programas e projetos educativos. A pesquisa registrou que os visitantes são, em sua maioria, jovens oriundos da Taquara e de outros bairros vizinhos; $81 \%$ do sexo masculino e $54 \%$ apresentando apenas o primeiro grau. $\mathrm{O}$ principal atrativo relatado pelos entrevistados é o banho de rio/cachoeira. O baixo nível de escolaridade da maioria dos visitantes precisa ser considerado na proposição de programas de Educação Ambiental voltados para UCs.

Palavras-chave: Unidade de conservação; turismo; perfil dos visitantes.

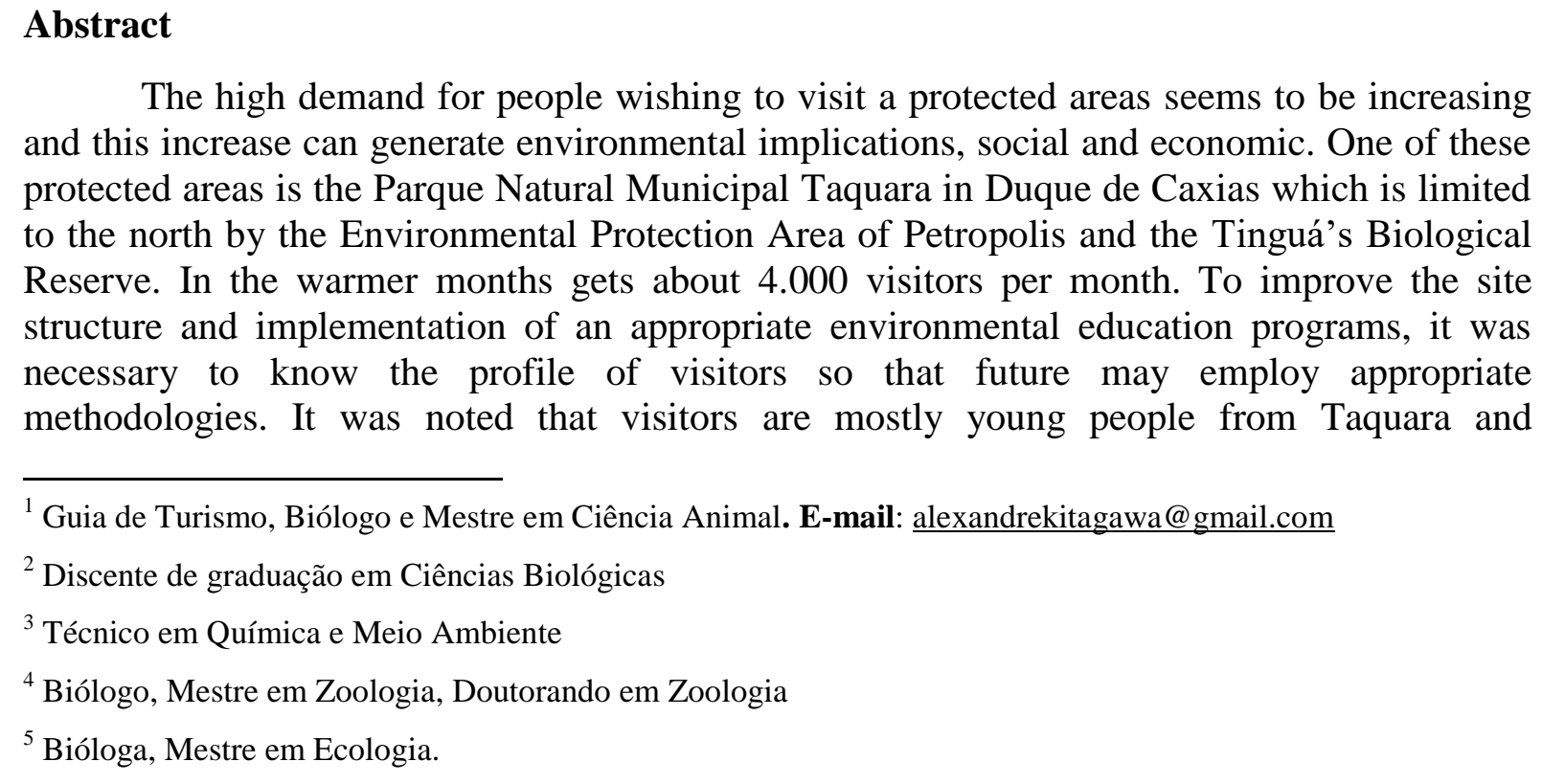

The high demand for people wishing to visit a protected areas seems to be increasing and this increase can generate environmental implications, social and economic. One of these protected areas is the Parque Natural Municipal Taquara in Duque de Caxias which is limited to the north by the Environmental Protection Area of Petropolis and the Tinguá's Biological Reserve. In the warmer months gets about 4.000 visitors per month. To improve the site structure and implementation of an appropriate environmental education programs, it was necessary to know the profile of visitors so that future may employ appropriate methodologies. It was noted that visitors are mostly young people from Taquara and

\footnotetext{
${ }^{2}$ Discente de graduação em Ciências Biológicas

${ }^{3}$ Técnico em Química e Meio Ambiente

${ }^{4}$ Biólogo, Mestre em Zoologia, Doutorando em Zoologia

${ }^{5}$ Bióloga, Mestre em Ecologia.
}

${ }^{1}$ Guia de Turismo, Biólogo e Mestre em Ciência Animal. E-mail: alexandrekitagawa@gmail.com 
surrounding neighborhoods, $81 \%$ are male, $54 \%$ had only primary school. The purpose of the trip was the most reported is swimming in the river/waterfall. The level of education can be a barrier to implementation of models of environmental education programs employed in other protected areas, and then to adapt them to the visiting public.

Keywords: Conservation unit; tourism; profile of visitors

\section{Introdução}

$\mathrm{O}$ incremento das atividades em áreas naturais protegidas pode gerar impactos (positivos e negativos) sociais, econômicos e ambientais. As Unidades de Conservação (UCs) são referências territoriais a serem consideradas no planejamento e gestão do uso público não predatório. Segundo Quintão (1983), o conceito de UCs modificou-se ao longo do tempo com o desenvolvimento científico, tecnológico e devido ao avanço acelerado das atividades humanas sobre os espaços naturais. No Brasil, a principal referência legal é a Lei 6.938/2000 que instituiu o Sistema Nacional de Unidades de Conservação da Natureza (SNUC). Na Lei, em seu artigo $2^{\circ}$, item I, as Unidades de Conservação (UCs) são definidas como:

“[...] espaço territorial e seus recursos ambientais, incluindo as águas jurisdicionais, com características naturais relevantes, legalmente instituído pelo Poder Público, com objetivos de conservação e limites definidos, sob regime especial de administração, ao qual se aplicam garantias adequadas de proteção;”.

O Parque Natural Municipal da Taquara (PNMT) é uma UC de proteção integral e possui aproximadamente 20 hectares, estando localizado no bairro da Taquara, $3^{\circ}$ distrito do Município de Duque de Caxias/RJ entre as coordenadas UTM - 0681274; 7499842 (SILVA et al., 2010). Limita-se ao Norte com a Área de Proteção Ambiental de Petrópolis e com a Reserva Biológica de Tinguá. Segundo Leite (2012), o PNMT recebe aproximadamente 4.000 visitantes por mês durante os meses mais quentes de verão.

O PMNT é parte integrante do Mosaico da Mata Atlântica Central Fluminense no Estado do Rio de Janeiro, sendo que esta última totaliza 233.710 hectares, abrangendo 13 municípios, 22 UCs e suas respectivas zonas de amortecimento (LINO; ALBUQUERQUE, 2007). Conflitos e problemas existem em grande parte das UCs do Rio de Janeiro e os investimentos em programas de Educação Ambiental (EA) poderão contribuir para amenizálos. Leite (2012), estudando a situação do PNMT, relacionou vários destes problemas, destacando a importância e a urgência de se investir em programas de EA nesses locais.

A proposta deste artigo se concentrou no levantamento do perfil dos visitantes do PMNT e estas informações poderão subsidiar a implantação de futuros programa de EA, assim como definir aspectos da infraestrutura necessária para funcionamento do parque.

\section{Material e métodos}

Os levantamentos foram realizados no mês de abril de 2013, sendo entrevistados 86 visitantes em dias aleatórios. Para obtenção dos dados foi utilizado um questionário prédefinido aplicado junto aos visitantes no portão de entrada do PNMT. O entrevistador 
perguntava e explicava os questionamentos quando estes se faziam necessários. As entrevistas consistiram de uma investigação contendo informações socioculturais, as atividades realizadas pelos visitantes, os aspectos mais positivos e negativos, a frequência de uso, entre outras. Posteriormente, os dados foram tabulados e analisados com o auxílio do software Microsoft Excel.

\section{Resultados e discussão}

Alguns parâmetros foram utilizados para estudar o perfil de visitantes da UC como: o grau de instrução, procedência, idade, intensidade de visitação, aspectos mais atrativos e atividade realizada. As consultas aos trabalhos de determinados autores serviram de base para esta orientação (FREITAS; MAGALHÃES; GUAPYASSÚ, 2002). Os resultados das entrevistas foram tabulados para melhor visualização.

a) Local de Residência

Para o Local de Residência, 48\% (41 pessoas) responderam que são do mesmo bairro, ou seja, moradores das adjacências e 52\% de outros bairros (Quadro I).

Quadro I. Local de residência dos visitantes entrevistados.

\begin{tabular}{|c|c|c|c|c|c|c|}
\hline \multicolumn{7}{|c|}{ LOCAL DE RESIDÊNCIA } \\
\hline Taquara & Pq. Paulista & Imbariê & St $^{\mathrm{a}}$.Lúcia & N.Campinas & St $^{\mathrm{a}}$.C.Serra & Outros \\
\hline $48 \%(41)$ & $12 \%(10)$ & $6 \%(5)$ & $2 \%(2)$ & $4 \%(3)$ & $7 \%(6)$ & $21 \%(18)$ \\
\hline
\end{tabular}

O resultado indica que grande parte dos visitantes mora no bairro da Taquara. Silva et al. (2010), também em estudo no PNMT afirmaram que $87 \%$ dos visitantes entrevistados eram moradores da Taquara ou bairros adjacentes. Fato semelhante ao que foi apresentado por Ribeiro e Cronemberger (2007) ao constatarem que os visitantes moradores de Petrópolis frequentam regularmente o Parque Nacional da Serra dos Órgãos (PARNASO). Em outras UCs como o Parque Nacional da Tijuca (PNT), em locais como Cascatinha, Meu Recanto e Paineiras, mais de 50\% dos visitantes são moradores das imediações (FREITAS, MAGALHÃES; GUAPYASSÚ, 2002).

A maior presença de visitantes de áreas adjacentes é explicado por Takahashi (1998), ao afirmar que o padrão natural na distribuição da procedência dos visitantes em áreas protegidas, reflete uma elevada frequência residentes das proximidades dos parques. Ribeiro e Cronemberger (2007) também afirmaram que os brasileiros não costumam viajar longas distancias para visitar uma UC.

Estes dados se mostram importantes, pois como afirmado por Freitas; Magalhães; Guapyassú (2002), a procedência dos visitantes é um importante indicador da inserção das áreas protegidas no contexto regional. 
b) Sexo dos visitantes

A amostragem no PMNT identificou que a grande maioria dos entrevistados era do sexo masculino, com $81 \%$ (Quadro II).

Quadro II. Sexo dos entrevistados.

\begin{tabular}{|c|c|}
\hline \multicolumn{2}{|c|}{ SEXO } \\
\hline Masculino & Feminino \\
\hline $81 \%(69)$ & $19 \%(16)$ \\
\hline
\end{tabular}

Silva et al. (2010), também encontrou um número maior de visitantes do sexo masculino $(60,0 \%)$ nas amostragens do PMNT. Em outras pesquisas feitas em UCs, como no Parque Nacional da Tijuca, houve ligeira diferença de homens $(54,39 \%)$ em relação às mulheres. Em parques estaduais de São Paulo, houve equilíbrio entre pessoas entrevistadas do sexo masculino $(50,7 \%)$ e feminino.

No Parque Estadual da Ilha Grande (PEIG), Araújo (2006) encontrou um resultado diferente, ou seja, 47,0\% de visitantes são do sexo masculino e 49,8\% do sexo feminino, sendo que $3,2 \%$ não opinaram. Apesar de, matematicamente, os dados sejam diferentes, estatisticamente talvez pode considerado como isento de diferença significativa.

Quanto aos turistas do Parque Estadual do Itapetinga em São Paulo, foram encontradas mais mulheres $(60,0 \%)$ do que homens (GONÇALVES; HOEFFEL, 2012). No Parque Estadual do Morro do Diabo (PEMD), também em São Paulo, Castro et al. (2007) constataram que $54,5 \%$ dos visitantes eram do sexo feminino.

A prática de atividades ao ar livre como rapel, trilhas, escaladas podem exercer maior atrativo às pessoas do sexo masculino, apesar de que muitas mulheres tem se envolvido, cada vez mais, nessas atividades. Deve-se considerar também o problema da segurança no interior das trilhas mais afastadas, o que pode limitar a presença mulheres. Esse assunto merece maior aprofundamento de pesquisa.

c) Estado Civil

Neste estudo notou-se que 54,0\% (46 pessoas) eram solteiras, 40,0\% (34 pessoas) eram casadas, 2,0\% (2 pessoas) divorciadas e $4 \%$ viúvos (3 pessoas) (Quadro III).

Quadro III. Estado civil dos visitantes entrevistados.

\begin{tabular}{|c|c|c|c|}
\hline \multicolumn{4}{|c|}{ ESTADO CIVIL } \\
\hline Solteiro & Casado & Divorciado & Viúvo \\
\hline $54 \%(46)$ & $40 \%(34)$ & $2 \%(2)$ & $4 \%(3)$ \\
\hline
\end{tabular}

No PEIG, Araújo (2006) relata que o número de visitantes solteiros $(65,5 \%)$ foi superior aos dos casados (23,0\%). Assim como no Parque Estadual Xixová-Japuí, São Paulo houve o predomínio de solteiros com 68,79\% e casados 26,3\% (MOURA et al., 2008). Niefer 
(2002), também constatou o predomínio do estado civil solteiro dentre os entrevistados (60,2\% - Ilha do Superagüi e 71,7\% - Ilha do Mel), e atribuiu ao acesso difícil e às vezes até perigoso, como sendo um dos motivos que impede uma maior visitação por famílias no Superagüi.

Esses dados parecem indicar que atividades em UCs, por um motivo ou por outro, são mais apreciadas pelo público solteiro.

d) Faixa etária

Houve uma grande diversidade de idades dentre os visitantes do PNMT, variando de pessoas com menos 18 até mais de 55 anos de idade (Quadro IV).

Quadro IV. Faixa etária dos visitantes entrevistados.

\begin{tabular}{|c|c|c|c|c|c|c|c|c|c|}
\hline \multicolumn{10}{|c|}{ FAIXA ETÁRIA } \\
\hline$<18$ & 18 a 20 & 21 a 25 & 26 a 29 & 30 a 35 & 36 a 40 & 41 a 45 & 46 a 50 & 50 a 55 & $>55$ \\
\hline $18 \%$ & $9 \%$ & $14 \%$ & $7 \%$ & $12 \%$ & $6 \%$ & $1 \%$ & $5 \%$ & $7 \%$ & $21 \%$ \\
$(15)$ & $(8)$ & $(12)$ & $(6)$ & $(10)$ & $(5)$ & $(1)$ & $(4)$ & $(6)$ & $(18)$ \\
\hline
\end{tabular}

Silva et al. (2010), também relatou que em seu estudo no PNMT, 13,0\% eram menores de idade e $87,0 \%$ maiores de idade. Valor muito próximo ao encontrado no presente estudo (18\% menor de 18 anos).

No Parque Estadual Xixová-Japuí, a faixa etária variou de 8 a 57 anos de idade, sendo que 53,6\% dos entrevistados tinham entre 15 e 24 anos (MOURA et al.,2008). No PNT, também há predomínio de visitantes jovens, que totalizam 65,7\% (MALTA e COSTA, 2009). O mesmo foi observado por em outras UCs, como o PARNASO por Ribeiro e Cronemberger (2007), onde 56,0\% dos entrevistados eram jovens. O interesse pelo público com esta faixa etária (mais jovens) pode estar relacionado com as atividades associadas a estes locais (trilhas, escalada, rapel, etc).

e) Grau de escolaridade

Quanto à escolaridade, os resultados mostraram que 54,0\% apresentavam o primeiro grau, seguidos de 33,0\% com segundo grau e 7,0\% com nível superior (Quadro V).

Quadro V. Grau de escolaridade dos visitantes entrevistados.

\begin{tabular}{|c|c|c|c|}
\hline \multicolumn{4}{|c|}{ ESCOLARIDADE } \\
\hline $1^{\circ}$ grau & $2^{\circ}$ grau & Graduação & Outros \\
\hline $54 \%(45)$ & $33 \%(28)$ & $8 \%(7)$ & $5 \%(4)$ \\
\hline
\end{tabular}

Moura et al. (2008) afirmou que do público que frequenta o Parque Estadual XixováJapuí, $43,5 \%$ possuem o $2^{\circ}$ Grau completo, seguido de $27,7 \%, 1^{\circ}$ Grau completo e $10,9 \%$ que possuem o nível superior (graduação). 
Já no PNT observou-se o inverso, com $61,4 \%$ dos visitantes apresentando nível de escolaridade superior, seguido de $29,8 \%$ dos indivíduos com o ensino médio e de 5,26\% visitantes que têm nível de escolaridade baixo, ou não concluiu o ensino fundamental (MALTA e COSTA, 2009). O mesmo pode ser observado no PARNASO, onde 59,0\% têm pelo menos o ensino superior completo (RIBEIRO e CRONEMBERGER, 2007), e no Parque Nacional de Itatiaia (PNI), onde 55,0\% possuem nível superior (BARROS, 2003).

O nível de escolaridade mais elevado supõe uma alta capacidade crítica, o que pode fazer com que exijam mais dos gestores e órgãos públicos, melhorando, assim a infraestrutura do local e absorvendo melhor os programas de Educação Ambiental implantados.

f) Finalidade da ida ao PNMT

Existem vários motivos para visitar uma UC. Dos propósitos da visita ao PNMT, o motivo mais citado foi o banho com 34\%, seguidos das outras finalidades (Quadro VI).

Quadro VI. Finalidade dos visitantes entrevistados para da ida ao PNMT.

\begin{tabular}{|c|c|c|c|c|c|}
\hline \multicolumn{5}{|c|}{ FINALIDADE } \\
\hline Caminhada & Banho & Estudo & Visitar morador & Morador & Outros \\
\hline $6 \%(5)$ & $34 \%(29)$ & $14 \%(12)$ & $14 \%(12)$ & $9 \%(8)$ & $22 \%(19)$ \\
\hline
\end{tabular}

No PARNASO, como no PNMT, os principais motivos das visitas são banho de rio ou cachoeira $(59,0 \%)$, percorrer uma trilha $(51,0 \%)$ e observar a fauna e a flora $(42,0 \%)$ (RIBEIRO e CRONEMBERGER, 2007).

Quando se compara os resultados obtidos no presente estudo com outros, observa-se que a finalidade muda de acordo com a UC. Malta e Costa (2009) em estudos no PNT observaram que 46,5\% dos indivíduos são "frequentadores", ou seja, utilizam-no para aliviar o "estresse", refletir, aproveitar as condições ambientais favoráveis para a prática de exercícios físicos, para o convívio social entre outras motivações. O mesmo ocorre com 30,9\% dos visitantes do Parque Estadual do Ibitipoca (PEIb), que afirmaram frequentar o parque pelos mesmos motivos. No PEMD, 72,0\% dos visitantes têm como finalidade realizar caminhadas (CASTRO et al., 2007).

Há um contraste muito grande quanto à finalidade da ida ao PNMT. No caso de outras UCs, em sua grande maioria os visitantes utilizam-na para realizar caminhadas. No PNMT percebe-se pelo vestuário que a finalidade principal não é realizar caminhadas, pois grande parte dos frequentadores trajava bermudas, chinelo e camiseta (sendo que uma parte não faz uso da camiseta), diferente dos trajes de quem realiza caminhada (trilhas). Isso também pode estar ligado a algum fator cultural, pois há relatos de moradores mais antigos que faziam o uso do rio como local de banho e lazer. 


\section{Conclusões}

O perfil dos visitantes do Parque Natural Municipal da Taquara é de pessoas que residem no mesmo bairro ou nas adjacências e que possuem nível de escolaridade relativamente baixo, quando comparado com a idade.

Os motivos que levam as pessoas ao Parque Natural Municipal da Taquara é quase sempre o banho de rio ou cachoeira, apesar de existirem placas sinalizando a proibição desta prática.

O baixo nível de escolaridade observado na amostragem deve ser considerado na montagem de programas de Educação Ambiental, regularmente empregados em outras Unidades de Conservação, havendo necessidade de adequá-los ao público visitante.

\section{Referências bibliográficas}

ARAÚJO, C.D. Perfil do visitante e capacidade de carga turística em Unidade de Conservação: o caso do Parque Estadual da Ilha Grande, RJ. 2006. 75 f. Dissertação (Mestrado em Ciências Ambientais e Florestais) - Universidade Federal Rural do Rio de Janeiro, Rio de Janeiro, 2006.

BARROS, M.I.A. Caracterização da visitação, dos visitantes e avaliação dos impactos ecológicos e recreativos do planalto do Parque Nacional de Itatiaia. 2003. $135 \mathrm{f}$.

Dissertação (Mestrado em Recursos Florestais) - Escola Superior de Agricultura Luiz de Queiroz, Universidade de São Paulo, Piracicaba, SP, 2003.

CASTRO, J. F. et al. O perfil dos visitantes do Parque Estadual do Morro do Diabo, Estado de São Paulo. In: CONGRESSO NACIONAL DE ECOTURISMO, 1., 2007. Anais... Rio de Janeiro, 2007.

FREITAS, W.R.; MAGALHÃES, L.M.S.; GUAPYASSÚ, M.S. Potencial de uso público do Parque Nacional da Tijuca. Acta Scientiarum, Maringá, v. 24, n. 6, p. 1833-1842, 2002.

LEITE, A. P. S. Parque Natural Municipal da Taquara: o que fazer para preservar. 2012. 38 f. Trabalho de Conclusão de Curso (Especialização) - Gestão Ambiental, AVM.

Faculdade Integrada, Rio de Janeiro. 38 fls. 2012.

LINO, C. F.; ALBUQUERQUE, J. L. Mosaicos de Unidades de Conservação no corredor da Serra do Mar. São Paulo: Conselho Nacional da Reserva da Biosfera da Mata Atlântica, 2007.

MALTA. R.R.; COSTA, N.M.C. Gestão do uso público em Unidade de Conservação: a visitação no Parque Nacional da Tijuca - RJ. Revista Brasileira de Ecoturismo, São Paulo, v.2, n.3, p. 273-294, 2009.

MOURA, C. de ET AL. Caracterização do perfil do visitante da praia de Itaquitanduva, Parque Estadual Xixová-Japuí, Baixada Santista, SP. IF Sér. Reg., São Paulo, n. 33, p. 1-11, 2008. 
NIEFER, I. A. Análise do perfil dos visitantes das Ilhas do Superagüi e do Mel: marketing como instrumento para um turismo sustentável. 2002. 237 f. Tese (Doutorado em Ciências Florestais)- Setor de Ciências Agrárias, Universidade Federal do Paraná, Curitiba,2002.

QUINTÃO, A.B. Evolução do conceito de Parques Nacionais e sua relação com o processo de desenvolvimento. Revista Brasil Florestal, Brasília, n. 54, p.13-28, 1983.

GONÇALVES, N. M.; HOEFFEL, J. L. M. Percepção ambiental sobre unidades de conservação: os conflitos em torno do Parque Estadual de Itapetinga - SP. Revista VITAS: Visões Transdisciplinares sobre Ambiente e Sociedade, n. 3, jun. 2012.

RIBEIRO, Tatiana Guillon ; CRONEMBERGER, Cecília. Perfil do visitante do Parque Nacional da Serra dos Órgãos. In: ENCONTRO INTERDISCIPLINAR DE ECOTURISMO EM UNIDADES DE CONSERVAÇÃO, 2., CONGRESSO BRASILEIRO DE ECOTURISMO, .6, 2007. Anais... Rio de Janeiro, 2007.

SILVA, N. C. dos S. et al. Consequências da atividade antrópica sobre as Unidades de Conservação estudo de caso: Parque Natural Municipal da Taquara, Duque de Caxias, RJ Brasil. In: CONGRESSO DE BIÓLOGOS DOS ESTADOS DO RIO DE JANEIRO E ESPÍRITO SANTO, 3., 2010. Anais... Rio de Janeiro, 2010. Resumos. 